\title{
Cervical malignant mixed mesonephric tumour: A case report with local recurrence after six-years and next-generation sequencing analysis with particular reference to the ataxia telangiectasia mutated gene
}

\author{
CARLA MARANI $^{1}$, IOLIA AKAEV ${ }^{2},{\text { CHIT CHENG } \text { YEOH }^{3}, \text { ELIZABETH WALSH }^{4} \text { and SIAVASH RAHIMI }}^{2,4}$ \\ ${ }^{1}$ Histopathology Division, San Carlo di Nancy Hospital, Rome 00165, Italy; ${ }^{2}$ School of Pharmacy and Biomedical Sciences, \\ University of Portsmouth, Portsmouth PO1 2UP; ${ }^{3}$ Department of Oncology, Queen Alexandra Hospital, \\ Portsmouth PO6 3LY; ${ }^{4}$ Frontier Pathology, Brighton and Sussex University Hospitals \\ NHS Trust, Royal Sussex County Hospital, Brighton BN2 5BE, UK
}

Received April 5, 2020; Accepted November 5, 2020

DOI: $10.3892 /$ etm.2021.9825

\begin{abstract}
Malignant mixed mesonephric tumours (MMMsT) of the female genital tract are extremely rare, and the majority are located in the wall of the cervix uteri. At present, there are no reports of the molecular characterisation of MMMsT of the female genital tract. Herein, we report the morphological, immunohistochemical and molecular features of this rare malignancy using next-generation sequencing (NGS) analysis. A 58-year-old woman presented with vaginal bleeding. In 2013, she had been diagnosed with a cervical carcinosarcoma of probable mesonephric origin and International Federation of Gynaecology and Obstetrics (FIGO) stage IB that had been treated by total hysterosalpingo-oopherectomy without adjuvant chemo-radiotherapy. Ultrasonography showed a vaginal mass measuring $25 \mathrm{~mm}$ in the maximum dimension. Biopsy was performed and showed a biphasic neoplasm composed of adenocarcinoma and sarcoma. Immunohistochemistry showed positive staining for epithelial membrane antigen (EMA), pancytokeratin (MNF116), paired box 8 (PAX-8), $\beta$-catenin, cytokeratin 7, cyclin D1, GATA3 and CD10. Androgen receptor positivity was detected in very limited areas. Cytokeratin 20, carcinoembryonic antigen (CEA), oestrogen receptor (ER), progesterone receptor (PR), transcription termination factor 1 (TTF1), Wilm's tumour antigen-1 (WT-1), calretinin and p16 were negative. The immunohistochemical profile was consistent with mesonephric origin. NGS analysis identified a variant of the ataxia-telangiectasia mutated (ATM)
\end{abstract}

Correspondence to: Dr Siavash Rahimi, Frontier Pathology, Brighton and Sussex University Hospitals NHS Trust, Royal Sussex County Hospital, Eastern Road, Brighton BN2 5BE, UK

E-mail: rahimi.siavash@gmail.com

Key words: mesonephric, malignant, mixed, female, cervix, ATM gene (p.Phe858Leu; c.2572 T>C; COSM21826). The number of detected allele frequency reads of ATM mutation following clinical relapse was higher, compared to its baseline: 65 vs. 96\%. The differential diagnosis of MMMsT includes mesonephric hyperplasia, malignant mixed Mullerian tumour (carcinosarcoma), endometrioid adenocarcinoma and endometrial stromal sarcoma. The clinical significance of the observed $A T M$ variant in the case reported herein is unknown. The present findings need further verification, as the mutation in ATM may result in chemotherapy resistance or conversely, may be exploited for targeted therapies.

\section{Introduction}

Mesonephric carcinoma of the female genital tract is a rare variant of adenocarcinoma that originates from the remnants of the mesonephric ducts (Wolffian ducts). Although the majority of cases arise in the wall of the cervix uteri, they may occur throughout the whole female genital tract $(1,2)$. Morphologically, they can be composed of a purely epithelial components (carcinoma) or include biphasic elements consisting of epithelial carcinomatous and mesenchymal sarcomatous components (1). This latter pathological entity, known as malignant mixed mesonephric tumour (MMMsT) or carcinosarcoma, is even less common than pure mesonephric carcinoma and is associated with worse prognosis than monophasic mesonephric carcinoma (1). Among mesonephric neoplasms of the female genital tract, cervical MMMsTs are exceedingly rare (3) and there is no report of the molecular characteristics of this condition. The present study reports a case of cervical MMMsT with full molecular characterisation by next-generation sequencing (NGS) analysis.

\section{Case report}

Clinical history and methods. In February 2019 a 58-year-old woman presented at San Carlo di Nancy Hospital (Rome, Italy) with a history of vaginal bleeding over several months. 
Gynaecological examination revealed a nodular mass in the posterior aspect of the vagina. Ultrasonography showed a solid mass with irregular borders measuring $25 \mathrm{~mm}$ in the maximum dimension. Her past medical history showed that, in July 2013 the patient had undergone hysterosalpingo-oophorectomy for a cervical carcinosarcoma of probable mesonephric origin and International Federation of Gynaecology and Obstetrics stage IB at San Giovanni Hospital (Rome, Italy). There was no history of other neoplasms. Chemotherapy and/or radiotherapy were not performed. A biopsy of the vaginal mass was taken. Slides of the original tumour were requested and only haematoxylin and eosin (H\&E) sections were made available for review. Resection of the vaginal recurrence, with clear margins, was performed.

Immunohistochemistry (IHC) was performed on formalin fixed paraffin embedded (FFPE) tissue blocks. Tissues were fixed in $10 \%$ neutral buffered formalin for $24 \mathrm{~h}$ at a room temperature. Sections of $3 \mu \mathrm{m}$ thickness were cut and stained on a BOND III Automated Immunohistochemistry System (Leica Biosystems) using a Leica Bond Polymer Refine Detection (Leica Biosystems; cat no. DS9800) and the antigen-unmasking solutions Epitope Retrieval Solution 1 (ER1) at pH 6.0 (Leica Biosystems; cat. no. AR9961) and Epitope Retrieval Solution 2 (ER2) at pH 9.0 (Leica Biosystems; cat. no. AR9640) for 20 min with the following antibodies: EMA (ready to use in ER1; mouse mAb clone GP1.4; Leica Biosystems; cat. no. PA0035), MNF116 (1:50 in ER2; mouse mAb clone MNF116; Dako; Agilent Technologies, Inc.; cat. no. M082101-2), cytokeratin 7 (1:50 in ER1; mouse mAb clone RN7; Leica Biosystems; cat. no. CK7-560-L-CE), cytokeratin 20 (1:400 in ER2; mouse mAb clone PW31; Leica Biosystems; cat. no. CK20-561-L-CE), PAX-8 (1:50 in ER2; mouse mAb clone MRQ-50; Cell Marque ${ }^{\mathrm{TM}}$; Sigma-Aldrich; Merck KGaA; cat. no. 363M-16), $\beta$-catenin (1:400 in ER1; rabbit pAb; Cell Signaling Technology, Inc.; cat. no. 9562S), cyclin D1 (1:40 in ER2; rabbit $\mathrm{mAb}$ clone SP4; Cell Marque ${ }^{\mathrm{TM}}$; Sigma-Aldrich; Merck KGaA; cat. no. 241R-16), GATA 3 (1:100 in ER2; mouse mAb clone L50-823; Cell Marque ${ }^{\mathrm{TM}}$; Sigma-Aldrich; Merck KGaA; cat. no. 390M-16), CD10 (1:50 in ER2; mouse mAb clone 55C6; Leica Biosystems; cat. no. CD10-270-L-CE), androgen receptor (1:200 in ER2; rabbit mAb clone EP120; Cell Marque ${ }^{\mathrm{TM}}$; Sigma-Aldrich; Merck KGaA; cat. no. AC-0071), p16 (1:10 in ER1; mouse mAb clone G175-405; BD Biosciences; cat. no. 550834), CEA (ready to use in ER2; mouse mAb clone COL-1; Leica Biosystems; cat. no. PA0364), ER (1:50 in ER1; mouse mAb clone 6F11; Leica Biosystems; cat. no. ER-6F11-L-F), PR (1:100; ER1; mouse mAb clone 16; Leica Biosystems; cat. no. PGR-312-L-F), TTF1 (ready to use in ER1; mouse mAb clone SPT24; Leica Biosystems; cat. no. PA0848), WT1 (ready to use in ER2; mouse mAb clone WT49; Leica Biosystems; cat. no. PA0562), calretinin (ready to use in ER2; mouse mAb clone CAL6; Leica Biosystems; cat. no. PA0346); p53 (ready to use in ER2; mouse mAb clone DO-7; Leica Biosystems; cat. no. PA0057) and Ki67 (ready to use in ER2; mouse mAb clone MM1; Leica Biosystems; cat. no. PA0230). A total of 20 slides were examined for H\&E and IHC. An upright light microscope (Olympus Corporation; model BX43) was used for examination of the stained slides.
Molecular analysis on both the primary and recurrent tumours was performed using a targeted semiconductor sequencing approach on an Ion Torrent Personal Genome Machine (PGM; Thermo Fisher Scientific, Inc.) (4). Tumour cellularity of the recurrent tumour (part 1, mainly sarcomatous) was estimated at $51-75 \%$, whereas the primary tumour (part 2, epithelial and sarcomatous) was at 5-20\%. Both samples were within assay acceptance criteria. FFPE histological sections were microdissected to include mainly tumour cells. Nucleic acid isolation was performed using a Gene Read FFPE DNA kit (Qiagen GmbH; cat. no. 180134). Extracted DNA was quantified using an Agilent 4200 TapeStation (Agilent Technologies, Inc.); thereafter, DNA samples were diluted to the desired concentration of $5 \mathrm{ng} / \mu \mathrm{l}$. The Ion Library Equalizer $^{\mathrm{TM}}$ Kit (Life Technologies; Thermo Fisher Scientific, Inc.; cat. no. 4482298) was used for library normalization at 100 pM. Tru-Q DNA Reference Standards (Horizon Discovery Group, plc; cat. nos. HD728, 729, 730 and 731) were included in the run.

The Ion AmpliSeq ${ }^{\mathrm{TM}}$ Cancer Hotspot Panel v2 (Life Technologies; Thermo Fisher Scientific, Inc.; cat. no. 4475346) and The Ion AmpliSeq ${ }^{\mathrm{TM}}$ Library Kit 2.0 (Life Technologies; Thermo Fisher Scientific, Inc.; cat. no. 4475345) were used to perform multiplex PCR for preparation of amplicon libraries ( 250 bp in length with $3^{\prime}$ P1 adapter incorporated by PCR) from genomic 'hot spot' regions. A panel covering 2,800 Catalogue Of Somatic Mutations In Cancer (COSMIC) mutations from 50 oncogenes and tumour suppressors, with the following genes, was used: $A B L 1, A K T 1, A L K, A P C, A T M$, $B R A F, C D H 1, C D K N 2 A, C S F 1 R, C T N N B 1, E G F R, E R B B 2$, ERBB4, EZH2, FBXW7, FGFR1, FGFR2, FGFR3, FLT3, GNA11, GNAQ, GNAS, HNF1A, HRAS, IDH1, IDH2, JAK2, JAK3, KDR, KIT, KRAS, MET, MLH1, MPL, NOTCH1, NPM1, NRAS, PDGFRA, PIK3CA, PTEN, PTPN11, RB1, RET, SMAD4, SMARCB1, SMO, SRC, STK11, TP53 and VHL.

Sequencing was performed on the PGM instrument using an Ion $\mathrm{PGM}^{\mathrm{TM}} \mathrm{Hi}^{\mathrm{-}} \mathrm{Q}^{\mathrm{TM}}$ View sequencing kit (Life Technologies; Thermo Fisher Scientific, Inc.; cat. no. A30044) with the Ion PGM $^{\text {TM }}$ Hi- $^{\text {TM }}$ View OT2 kit (Life Technologies; Thermo Fisher Scientific, Inc.; cat. no. A29900) and run on an Ion $318^{\mathrm{TM}}$ Chip Kit v2 BC (Life Technologies; Thermo Fisher Scientific, Inc.; cat. no. 4488150). For mutation analysis, Torrent Suite v5.0.5 and Variant Caller v5.0.4.0 (Life Technologies; Thermo Fisher Scientific, Inc.) were used, followed by variant annotation in Ion Reporter v5.10 software (Life Technologies; Thermo Fisher Scientific, Inc.) with custom filters and functional characterization via MutationTaster (http://www. mutationtaster.org) and Varsome (https://varsome.com) to group the Variant Caller output into the reported categories (single-nucleotide variant and small indels). Variant descriptors were reported according to Human Genome Variation Society recommended nomenclature with the corresponding COSMIC reference number. The patient did not consent for matched normal sample and/or blood molecular analysis, due to the family implications of germline mutation testing.

\section{Results}

Histological examination of the original tumour and recurrent mass demonstrated a biphasic neoplasm composed of 

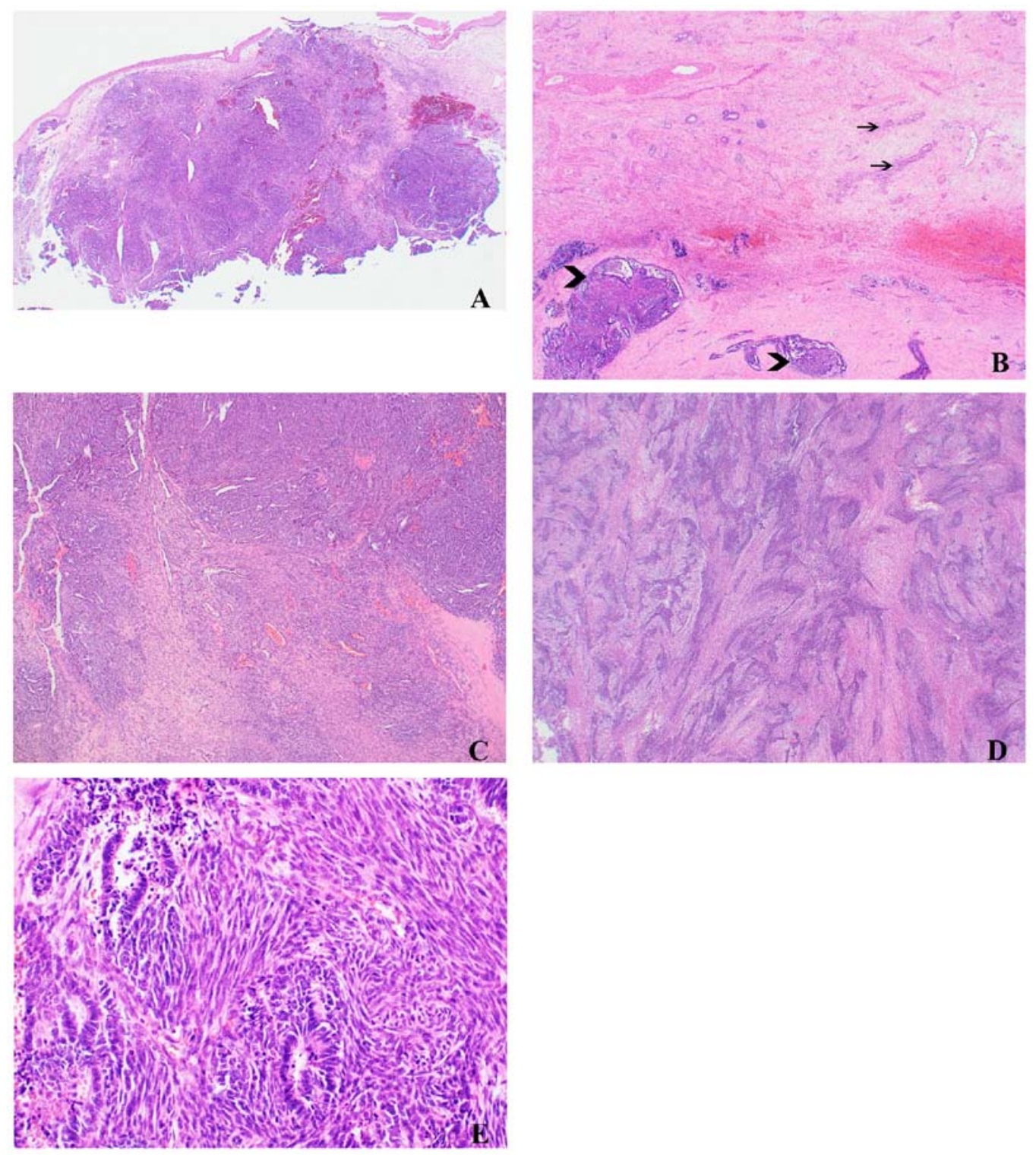

Figure 1. (A) Low-magnification (x2) examination of the recurrent tumour in the vagina showing a biphasic neoplasm. (B) Low magnification (x2) examination of the original cervical neoplasm showing islands of MMMsT (arrowheads). Foci of mesonephric hyperplasia are identified (arrows). (C) Original cervical MMMsT showing complex glandular architecture (x10 magnification). (D) Sarcomatous component of the vaginal tumour shows alternating hypocellular and hypercellular areas (x10 magnification). (E) Higher magnification (x40) showing abrupt transition between glandular and sarcomatous components. MMMsT; malignant mixed mesonephric tumour.

malignant epithelial adenocarcinomatous and mesenchymal sarcomatous components (Fig. 1A). The original neoplasm presented infiltrative margins and had arisen in the background of mesonephric hyperplasia (Fig. 1B) that consisted of clusters of small/medium-sized tubules lined by bland cuboidal cells, with no nuclear atypia and/or pleomorphism. Most of the tubule lumens contained a densely eosinophilic secretion. The recurrent tumour did not contain foci of benign mesonephric remnants.

The primary and recurrent tumour showed similar morphological features. However, the original tumour contained a higher proportion of carcinomatous components while the recurrent tumour contained a higher proportion of sarcomatous components. The epithelial components displayed a variety of architectural patterns, including tubular, solid, papillary, retiform and ductal (glandular) elements (Fig. 1C), which were lined by a single row of cuboidal cells, with moderate atypia. Occasional pseudo-papillae and slit-like spaces, reminiscent of serous carcinoma, were present. Some tubules contained eosinophilic secretions. In the primary tumour, the tubules infiltrated and dissected the cervical wall in a random, hazardous manner. The mesenchymal sarcomatous component displayed alternating hypocellular areas with dense, hypercellular areas (Fig. 1D) and was composed of cells with spindle-cell nuclei and scanty basophilic cytoplasm, arranged in short intersecting fascicles. There was moderate nuclear atypia and frequent mitoses. Myxoid changes were present and no heterologous elements were identified. The transition between epithelial and mesenchymal components was abrupt (Fig. 1E). Mitotic figures, in both components, were frequent, $\sim 12$ per 10 high power fields (HFP). No necrosis was identified. There was no lymphovascular invasion.

Immunohistochemistry demonstrated diffuse positive staining of EMA, MNF116, PAX-8 (Fig. 2) and $\beta$-catenin (membranous). 

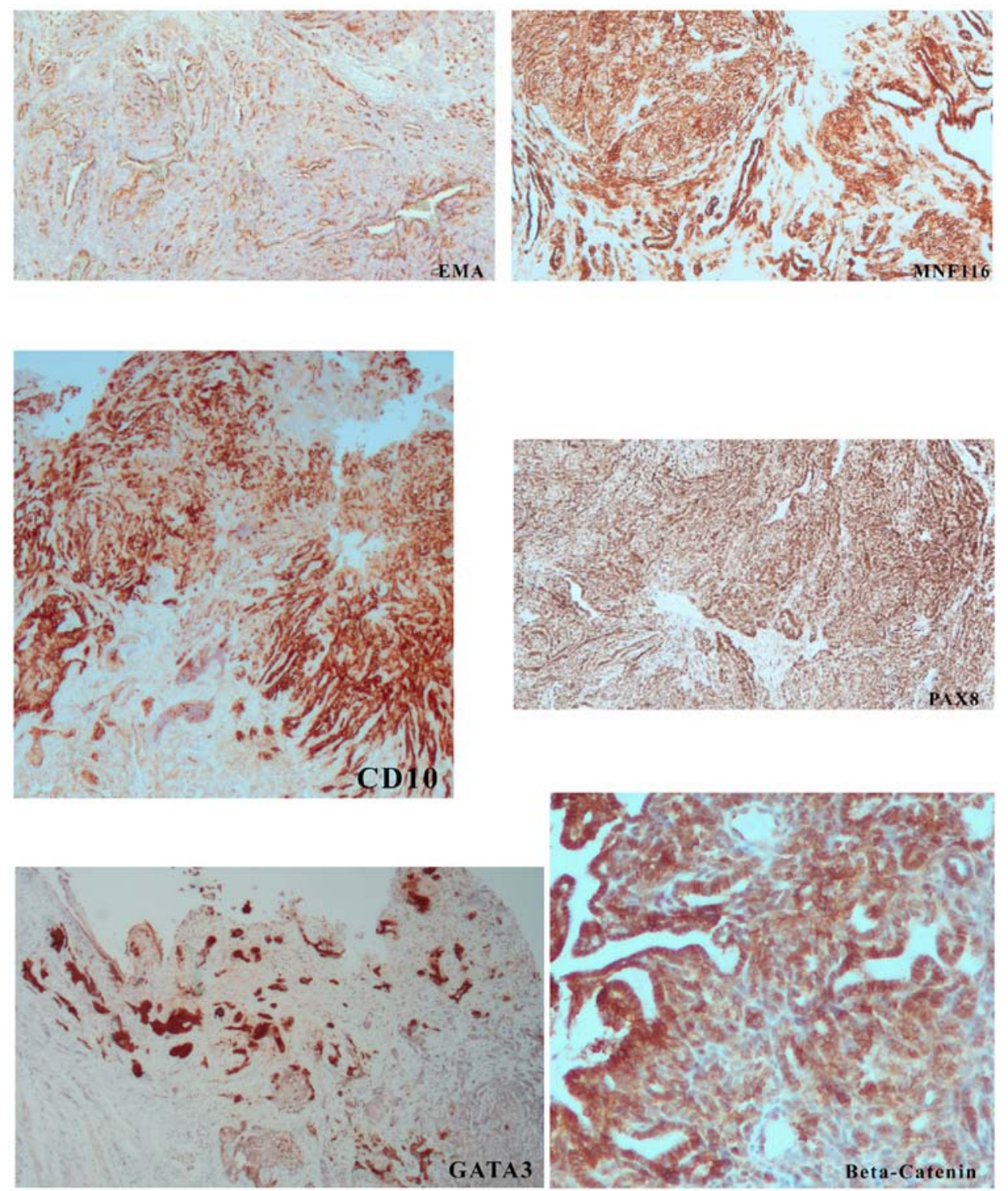

Figure 2. Positive immunostaining of EMA, pancytokeratin MNF116, CD10, PAX-8, GATA3 and $\beta$-catenin (all magnifications x4). EMA, epithelial membrane antigen; PAX-8, paired box 8 .

Cytokeratin 7 and cyclin D1 were present in $\sim 15$ and $40 \%$ of lesional cells, respectively. GATA 3 and CD10 (apical/luminal) were positive in $\sim 60 \%$ of cells (Fig. 2). Androgen receptor positivity was detected in $~ 5 \%$ of cells. p16 showed a patchy positivity with no 'block-type' pattern. Cytokeratin 20, monoclonal CEA, ER, PR, TTF1, WT1 and calretinin were negative. p53 showed normal levels of expression. Ki67 staining, a marker of proliferation, showed positive nuclear staining in $\sim 70 \%$ of the lesional cells. Positive CD10 and GATA3 and negative p16 immunostaining were consistent with a mesonephric origin.

No mutations with currently established therapeutic implications were detected either in primary or recurrent samples. In particular, there were no mutations in TP53, BRAF, EGFR, $K R A S, N R A S$ and $C T N N B 1$. However, a missense variant of $A T M$ (p.Phe858Leu, c.2572 T>C, COSM21826)-[Sequence Ontology: SO:0001583], was observed in $65 \%$ of the sequencing reads in the original tumour and in $96 \%$ of the sequencing reads in the recurrent tumour.
The patient is alive and well without recurrence one year after surgery. No additional treatment was administered.

\section{Discussion}

Cervical malignant mesonephric tumours are rare, and the majority are adenocarcinomas (1). MMMsTs are extremely rare and, therefore, understudied. To the best of our knowledge, only 11 cases of MMMsTs have been reported (3). MMMsTs are also called carcinosarcomas; however, this may be an improper term, as it may evoke a Mullerian tract derived neoplasm. Mesonephric tumours arise from mesonephric remnants that are usually found in the lateral walls of the cervix and less frequently adjacent to the ovarian hilum, in the broad ligament and in the vagina (1). The prevalence of mesonephric remnants varies from $1-22 \%$ in adults and up to $40 \%$ in children $(5,6)$.

The morphological and immunohistochemical data of the present case are consistent with those of previous reports (3). The diagnosis of cervical MMMsTs from resection specimens 
should not be arduous, as the neoplasm arises in the context of mesonephric hyperplasia and often the background of benign mesonephric remnants is present. Diagnosis from biopsy samples, however, may be problematic. If only the epithelial component is present, differential diagnosis with florid mesonephric hyperplasia can be difficult. Morphological features such as atypia, brisk mitotic activity and the aforementioned architectural pattern are helpful criteria. The rate of proliferation, assessed by $\mathrm{Ki67}$, is also a useful diagnostic clue (7). Cervical mixed Mullerian malignant tumour (carcinosarcoma) is also included in the differential diagnosis. However, this neoplasm may show squamous differentiation that is not present in MMMsTs. Endometrioid endometrial adenocarcinoma, that extends into the cervix, may show mesonephric morphological features (8). The $\mathrm{CD}^{+} 0^{+}, \mathrm{ER}^{-}$and $\mathrm{R}^{-}$immunoprofile of MMMsTs distinguishes them from an endometrioid carcinoma. When a sarcomatous component is present, the differential diagnosis is with endometrial stromal sarcoma (ESS). MMMsTs do not exhibit the typical vasculature pattern of ESS, as low-grade ESS is $\mathrm{ER}^{+}$and $\mathrm{PR}^{+}$. In high-grade MMMsTs, molecular analysis can rule out ESS (9).

Data on the molecular features of mesonephric malignancies of the female genital tract, and MMMsT in particular, are very limited. Mesonephric carcinomas with no sarcomatous component have been characterised at the molecular level using NGS (10). A total of $81 \%$ of cases had a KRAS or NRAS mutation, while mutations in ARIDIA, ARIDIB or SMARCA4 (chromatin remodelling genes) were identified in $62 \%$ of cases. In $75 \%$ of cases, a copy number alteration (1q gain) was identified. No mutations in PIK3CA and PTEN were present. Based on these findings the authors concluded that the molecular aberrations in mesonephric carcinomas differed from cervical and endometrial adenocarcinoma, which displayed $K R A S$ and $N R A S$ mutations in 7 and $25 \%$ of cases, respectively.

The ATM protein belongs to the phosphatidylinositol 3-kinase (PIK3) family of proteins that participate in DNA repair and encodes a protein kinase that phosphorylates multiple targets following double stranded breaks; it plays a role in cell cycle checkpoint arrest, DNA repair and apoptosis (11). Germline mutations in ATM result in ataxia telangiectasia syndrome (ATS), and patients with ATS show increased cancer predisposition, primarily development of lymphomas or leukaemia $(12,13)$. Sporadic ATM mutations are relatively common in haematological malignancies, as well as in a wide spectrum of tumours, including breast, pancreatic and lung cancers $(12,14)$.

To the best of the authors' knowledge, ATM mutations in the female genital tract mesonephric neoplasms (adenocarcinoma and MMMsT) have not been reported. The results of NGS analysis in the case reported herein are interesting; however, they should be interpreted with great caution. In the primary tumour the missense mutation was seen in $65 \%$ of the sequencing reads, and may be the result of either germline or somatic mutation. The same ATM variant was found in $96 \%$ of the recurrent tumour DNA. In a scenario with a single clone of cells, we would expect possibilities of finding $0 \%$ mutation, $50 \%$ (one allele inactivated) or 100\% (both alleles inactivated), unless there was a gene amplification. Our results of between 50 and $100 \%$ suggest that the cell population is not monoclonal and includes stromal and inflammatory cells or consists of a different proportion of heterozygous and homozygous mutated cells, either germline or somatic. However, considering the difference in the proportion of the tumour epithelial and sarcomatous components, and the tumour cellularity in both samples, with the sarcomatous component being predominant in the recurrent tumour, the higher value of $96 \%$ may be associated with the expansion of one clone. Nevertheless, $96 \%$ is very close to the assumed homozygous germline mutation rate of $100 \%$. Therefore, an artefactual effect due to excessive formalin fixation of the specimen cannot be excluded. The possibility that the same presumed somatic ATM variant detected in the primary neoplasm was also identified in the recurrent tumour cannot be ruled out. As the patient did not consent for normal tissue and blood molecular analysis, a germline mutation cannot be excluded.

There is no clear evidence that this missense variant (p.Phe858Leu) results in loss of function of the ATM gene. Both germline and somatic mutations span the entire ATM gene and occur in each functional domain (11). This heterogeneity makes it challenging to make firm predictions of the clinical consequences. Therefore, this variant would be considered of uncertain clinical significance with respect to the pathogenesis of MMMsTs. In terms of clinical consequences, analysis of breast cancer and control cases by Stredrick et al (15) concluded that the p.Phe858Leu, c.2572T >C missense variant was seen at $\sim 2 \%$ frequency and was associated with a significantly increased risk of breast cancer in the USA, but not in Poland (15). Estiar and Mehdipour (16) reported in their comprehensive review on breast and brain tumour ATM mutational studies, that the c.2572T $>$ C mutation was mainly detected in breast, but not in brain tumours. Additionally, the same mutation was found in two endometrial carcinoma samples reported in the COSMIC database by Rosa-Rosa et al (17) but was not considered pathogenic.

The ATM mutation p.(Phe858Leu), c.2572 T>C (http://www.ncbi.nlm.nih.gov/clinvar/variation/132736/), has been most commonly reported as benign, likely-benign or conflicting-interpretations-of-pathogenicity $(18,19)$. However, in view of the results of the case reported herein, the involvement of this variant in the pathogenesis of MMMsT cannot be ruled out. Therefore, investigation into mutations in the ATM gene should be considered in further suspected cases of MMMTs.

\section{Acknowledgements}

Not applicable.

\section{Funding}

No funding was received.

\section{Availability of data and materials}

The datasets used and/or analysed during the current study are available from the corresponding author on reasonable request.

\section{Authors' contributions}

All the authors were involved in conceiving and designing the present study. CM designed, drafted and wrote the manuscript. IA, CCY and EW were responsible for collecting and analysing 
patient data. SR designed, drafted the manuscript and revised it critically for important intellectual content. All authors have read and approved the final version of the manuscript.

\section{Ethics approval and consent to participate}

Signed informed consent was obtained from the patient.

\section{Patient consent for publication}

The patient gave signed consent for publication; however, the authors also made efforts to remove any identifying information to protect the privacy of the patient.

\section{Competing interests}

The authors declare that they have no competing interests.

\section{References}

1. Howitt BE and Nucci MR: Mesonephric proliferations of the female genital tract. Pathology 50: 141-150, 2018.

2. Yano M, Shintani D, Katoh T, Hamada M, Ito K, Kozawa E, Hasegawa $\mathrm{K}$ and Yasuda M: Coexistence of endometrial mesonephric-like adenocarcinoma and endometrioid carcinoma suggests a Müllerian duct lineage: A case report. Diagn Pathol 14: 54, 2019.

3. Ribeiro B, Silva R, Dias R and Patrício V: Carcinosarcoma of the uterine cervix: A rare pathological finding originating from mesonephric remnants. BMJ Case Rep 12: e227050, 2019.

4. Exome Sequencing by Ion Torrent Next-Generation Sequencing. Thermo Fisher Scientific, UK, 2020; Accessed July 19, 2020; Available from: https://www.thermofisher.com/uk/en/home/ life-science/sequencing/dna-sequencing/exome-sequencing/exomesequencing-ion-torrent-next-generation-sequencing.html.

5. Cavalcanti MS, Schultheis AM, Ho C, Wang L, DeLair DF, Weigelt B, Gardner G, Lichtman SM, Hameed M and Park KJ: Mixed mesonephric adenocarcinoma and high-grade neuroendocrine carcinoma of the uterine cervix: Case description of a previously unreported entity with insights into its molecular. Int J Gynecol Pathol 36: 76-89, 2017.
6. Ferry JA and Scully RE: Mesonephric remnants, hyperplasia, and neoplasia in the uterine cervix. A study of 49 cases. Am J Surg Pathol 14: 1100-1111, 1990.

7. Jimenez C, Nucci M and Zaloudek C: Mesonephric adenocarcinomas of the uterus and cervix - A clinicopathologic study of 10 cases. Mod Pathol 25: 278A, 2012.

8. Tambouret R, Clement PB and Young RH: Endometrial endometrioid adenocarcinoma with a deceptive pattern of spread to the uterine cervix: A manifestation of stage IIb endometrial carcinoma liable to be misinterpreted as an independent carcinoma or a benign lesion. Am J Surg Pathol 27: 1080-1088, 2003.

9. Lee $\mathrm{CH}$ and Nucci MR: Endometrial stromal sarcoma - the new genetic paradigm. Histopathology 67: 1-19, 2015.

10. Mirkovic J, Sholl LM, Garcia E, Lindeman N, MacConaill L, Hirsch M, Dal Cin P, Gorman M, Barletta JA, Nucci MR, et al: Targeted genomic profiling reveals recurrent KRAS mutations and gain of chromosome $\mathrm{lq}$ in mesonephric carcinomas of the female genital tract. Mod Pathol 28: 1504-1514, 2015.

11. Shiloh Y: ATM: Expanding roles as a chief guardian of genome stability. Exp Cell Res 329: 154-161, 2014.

12. Choi M, Kipps T and Kurzrock R: ATM mutations in cancer: Therapeutic implications. Mol Cancer Ther 15: 1781-1791, 2016.

13. Abdolrahimzadeh S, Plateroti AM, Recupero SM and Lambiase A: An update on the ophthalmologic features in the phakomatoses. J Ophthalmol 2016: 3043026, 2016.

14. Forbes SA, Beare D, Gunasekaran P, Leung K, Bindal N, Boutselakis H, Ding M, Bamford S, Cole C, Ward S, et al: COSMIC: Exploring the world's knowledge of somatic mutations in human cancer. Nucleic Acids Res 43: D805-D811, 2015.

15. Stredrick DL, Garcia-Closas M, Pineda MA, Bhatti P, Alexander BH, Doody MM, Lissowska J, Peplonska B, Brinton LA, Chanock SJ, et al: The ATM missense mutation p.Ser49Cys (c.146C $>$ G) and the risk of breast cancer. Hum Mutat 27: 538-544, 2006.

16. Estiar MA and Mehdipour P: ATM in breast and brain tumors: A comprehensive review. Cancer Biol Med 15: 210-227, 2018.

17. Rosa-Rosa JM, Leskelä S, Cristóbal-Lana E, Santón A, López-García MA, Muñoz G, Pérez-Mies B, Biscuola M, Prat J, Esther $\mathrm{O}$, et al: Molecular genetic heterogeneity in undifferentiated endometrial carcinomas. Mod Pathol 29: 1390-1398, 2016.

18. 11-108137983-108138023 I gnomAD. Retrieved July 20, 2020; Available from: https://gnomad.broadinstitute.org/region/11-108 137983-108138023?dataset=gnomad $\mathrm{r} 2$ 1.

19. VCV000132736: 10 - ClinVar - NCB̈I. Retrieved July 22, 2020; Available from: https://www.ncbi.nlm.nih.gov/clinvar/ variation/132736/. 